\title{
ACTIVE TILT CONTROL OF BRAKE DISC BOLT LOAD FOR HEAVY SEMITRAILER
}

\author{
Hao Wang ${ }^{1}$ \\ ${ }^{1}$ Department of Automotive Engineering, Henan Polytechnic Institute, Henan Province, 473000, China. \\ Email: wanghaowh2012@yeah.net
}

\begin{abstract}
In order to explore the active roll control of brake disc bolt load of heavy semi-trailer, the finite element simulation model of brake disc is constructed by using thermal coupling simulation algorithm, vehicle friction braking principle and heat transfer principle, and the temperature field and thermal stress bolt load of brake disc are analyzed. The results show that when analyzing the internal temperature of the brake disc, the heat transfers from the disc to the inner side, the temperature of the disc first rises and then decreases, and the internal temperature keeps rising. When analyzing the finite element model of the brake disc, it is found that during the braking process, the tensile stress and equivalent stress on the upper side of the bolt hole in the tooth of the brake disc are the largest parts, which are most vulnerable to damage. Therefore, through the research on active roll control of brake disc bolt load of heavy semi-trailer, the finite element simulation model is validated to meet the expectations. Although there are some shortcomings in the research process, it still provides some new ideas for the improvement of brake disc of heavy semi-trailer.
\end{abstract}

Keywords: Heavy Semi-Trailer; Brake Disc; Bolt Load; Finite Element Model.

\section{Introduction}

Today, with the development of society, with the progress of scientific level, online shopping and other behaviors are increasing. In order to make people get their own parcels faster, logistics efficiency is increasing. As the main transport vehicle, heavy semi-trailer has already accounted for more than half of the country's road freight volume, and in the 13th Five-Year Plan, heavy semi-trailer has been included in one of the eight major tasks of transportation development $[1,2]$. Due to the characteristics of heavy semi-trailer, such as large weight and volume, high centric position and narrow wheelbase relative to body height, the most prominent problem at low speed is turning maneuverability, or named path following [3].

Its own characteristics lead to heavy wear of semi-trailer tires and damage to road surface when turning. It is also worth paying attention to the potential safety hazards caused by the wide passageway to the surrounding facilities, pedestrians and vehicles. The most prominent problems at high speed include tail swing, folding and rollover, which are one of the most important causes of many serious traffic accidents, causing huge economic losses and casualties [4].

In order to solve the turning maneuverability of heavy semi-trailer at low speed, the wear of semitrailer tires, the occupancy width, and the risk of damage to the surrounding environment (people, vehicles, roads), are reduced by widely using the passive and active steering system of semi-trailer. The former is more widely used because of its low cost. Its hardware strategies include electronic control braking system (ESC) of semi-trailer, electronic height control module (ELM) of air suspension and other active/semi-active suspension systems, active lateral stabilizer, semi-trailer active steering system and variable damping fifth wheel system [5].

These systems can prevent the heavy semi-trailer from providing additional yaw moment when the high-speed instability occurs, restrain the tail-flick of the semi-trailer, reduce the center of gravity, and restrain the roll of a vehicle by using the elastic force of the elastic rod. These systems can also improve vehicle mobility and stability; restrain the folding of two body units, and so on [6]. As a fastener connecting two or more objects, bolts have problems of friction, contact and tightening, which will make the dynamic characteristics more complex. In addition, the stress will change complex, and the structural reliability may be insufficient [7]. During the whole braking process, the friction between the brake disc and the brake pad will produce huge heat, most of which will be absorbed by the brake disc, which will lead to a sharp rise in the temperature of the brake disc, and then generate thermal expansion.

The bolt will also be stretched to produce tensile stress [8]. If the stress value exceeds the strength limit of the bolt, the bolt will be pulled off, the brake 
disc will fall off the wheel, and the brake will completely fail, resulting in serious consequences. In order to improve the high-speed stability of heavy semi-trailer, a lot of theoretical and experimental studies have been carried out on the load performance of bolts in brake discs at home and abroad.

In conclusion, in order to further clarify the reliability of some brake disc bolts currently used in heavy semi-trailers, understand the load characteristics and damage rules of bolts; the finite element simulation model is constructed by thermal stress coupling method.

The temperature field of brake disc is calculated by energy conversion method and friction power method, and the load of thermal stress bolt is analyzed, so as to provide reference for the improvement of heavy semi-trailer.

\section{Methodology}

\subsection{Load analysis method of brake disc bolt}

Rake disc damage is mainly due to excessive local stress caused by external loads. The train external load is mainly composed of thermal load in braking process and centrifugal force, road spectrum vibration and shock in high-speed driving process. In braking process, the change of temperature field and stress field of brake disc has great influence on its mechanical properties. Coupling analysis of temperature field and structural physical field can provide theoretical basis for mechanical checking and fatigue damage of brake disc [9].

In recent years, the finite element method (FEM) has been widely used to study the temperature and stress fields of some materials and structures at home and abroad. The load calibration curve of a bolt is shown in figure 1 .

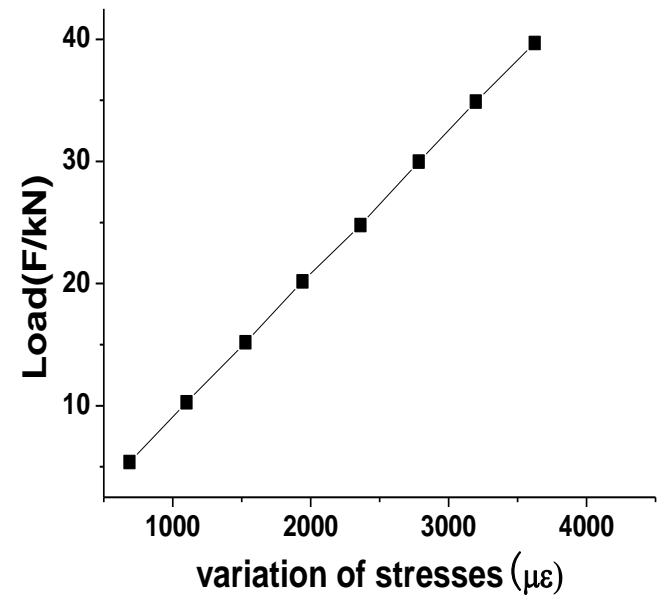

Figure 1: Calibration curve of bolt load

According to the load conditions of the brake disc under various working conditions, there are roughly two kinds of stress sources leading to the damage of the brake disc. One of them is that in the process of vehicle friction braking, the friction between brake disc and brake pad produces a huge thermal load, which causes the deformation of the brake disc and thermal stress in the deformation part. The other is that in the process of high-speed train operation, the wheel-axle system is subjected to road spectrum vibration. Additional vertical acceleration is produced by dynamic and impact, and centrifugal force is produced when wheel axle drives brake disc to rotate at high speed. The combined action of the two forces will cause dynamic stress and cyclic alternating stress of brake disc [10]. Therefore, the mechanism of bolt load damage in brake disc from the aspect of braking will be analyzed.

\subsection{Thermal stress coupling method of brake disc}

Before braking, the brake disc is at room temperature. After braking, when studying the temperature field of brake disc under friction braking condition, the parameters such as friction coefficient and pressure on the disc surface cannot be ignored, and these parameters are related to the contact condition of the disc surface, so the key to study the temperature field is to choose a reasonable contact model. Brake disc is a complex multiphysical field coupling process in train braking process, and there are many factors affecting the temperature field and stress field. Thermalstructural coupling analysis methods are mainly divided into direct coupling method and sequential coupling method. The direct coupling method is to directly establish the contact model and analyze the coupled element with displacement and temperature freedom. The temperature field and stress field can be obtained simultaneously [11]. The sequential coupling method divides thermal analysis and structural stress analysis into two steps. Firstly, the temperature of each node is calculated, and then applied to the structure as a known body load.

At present, the sequential coupling method is widely used in the analysis of thermal-structural coupling field of brake disc braking process.

Vehicle braking process is a process of transforming kinetic energy into heat energy, which can be analyzed according to the law of energy conservation. It is assumed that all kinetic energy is converted into heat energy in this process. The heat generated during the friction braking process is as follows.

$$
Q(t)=W=\frac{1}{2} M\left(v_{0}^{2}-v_{t}^{2}\right)
$$

In the formula, M - axle load, kg; V0 - initial speed of train friction braking, m / s; VT - instantaneous speed of braking t seconds, $\mathrm{m} / \mathrm{s}$.

Considering many factors affecting the actual braking process, only part of the kinetic energy of the vehicle is converted into heat energy absorbed 
by the brake disc, so the energy conversion rate is introduced. In addition, in actual braking, each axle is equipped with multiple brake discs, that is, the heat generated by friction is absorbed by multiple brake discs, and so the input heat of each disc can be corrected as follows.

$$
Q(t)=\eta \frac{W}{n}
$$

In the formula, $\eta$ is the percentage of the total energy absorbed by the brake disc. $\mathrm{n}$ is the number of friction surfaces on each axle.

The specific analysis process of thermalstructural coupling is to establish a threedimensional finite element model of the brake disc, establish the boundary conditions of the thermal input and output of the brake disc, and then conduct thermal analysis of the brake disc. The node temperature obtained by solving the temperature field is loaded on the model of structural analysis, and the effect of centrifugal force in braking process is fully considered to complete the thermal stress field analysis.

\subsection{Finite element model of brake disc stress}

Because of the braking process, the temperature of the brake disc increases due to the action of huge thermal load. The thermal conductivity and specific heat capacity of the material will change with the increase of temperature.

Therefore, the material nonlinearity should be fully considered in the thermal analysis of brake discs. Thermal material properties of the brake disc are shown in table 1.

Table 1. Relation table of thermal performance parameters of brake disc material with temperature

\begin{tabular}{|c|c|c|c|}
\hline $\begin{array}{c}\text { Temperature } \\
{ }^{\circ} \mathrm{C}\end{array}$ & $\begin{array}{c}\text { Thermal } \\
\text { conductivity } \\
\lambda \mathrm{W} /(\mathrm{m} . \mathrm{K})\end{array}$ & $\begin{array}{c}\text { Specific } \\
\text { heat } \\
\text { capacity } \\
\mathrm{c} \\
\mathrm{J} / \mathrm{kg} \cdot \mathrm{K})\end{array}$ & $\begin{array}{c}\text { Density } \\
\rho\left(\mathrm{kg} . \mathrm{m}^{-}\right. \\
3)\end{array}$ \\
\hline 25 & 38.2 & 520 & 7850 \\
\hline 100 & 47.6 & 523 & 7850 \\
\hline 200 & 46.2 & 536 & 7850 \\
\hline 300 & 43.5 & 565 & 7850 \\
\hline 400 & 41.3 & 578 & 7850 \\
\hline 500 & 38.5 & 571 & 7850 \\
\hline
\end{tabular}

\subsection{Hypothetical conditions for thermal analysis of brake discs}

The brake disc is heated by friction of the brake pad during braking. At the same time, due to the role of thermal radiation and heat convection, the brake disc continuously releases heat to the surrounding air.
According to the theory of friction braking and temperature field analysis, the following assumptions are made for braking process.

Firstly, the braking process of the train is a uniform deceleration movement. The brake pad pressure is constant and the friction coefficient is constant.

Secondly, the brake pad and the brake disc are in face contact, and the contact pressure on the contact surface is uniformly distributed.

Thirdly, the heat generated by friction during braking is fed to the friction surface of the brake disc in the form of heat flow.

Fourthly, heat convection and radiation are the main heat dissipation in braking process, while heat transfer between disc and other structures is neglected.

Fifthly, when the brake disc rotates around a circle, the thermal loads at all points of the brake disc in the same radial position are equal.

\subsection{Establishment of boundary model for thermal analysis of brake disc}

The heat generated by friction is applied to both sides of the brake disc in the form of heat flux.

According to the energy folding algorithm, the heat flux formula on the friction surface determined by the energy folding algorithm is as follows:

$$
q(t)=-\eta \frac{M a\left(v_{0}+a t\right)}{n S}
$$

The formula of heat flux on friction surface determined by friction power method is as follows:

$$
q(t, r)=-\eta \frac{\operatorname{Mar} \varphi_{r}\left(v_{0}+a t\right)}{n r_{f r i} \cdot 2 \pi S_{p}}
$$

In the formula, the friction radius $r_{f r i}$ is $0.145 \mathrm{~m}$ and the brake pad $S_{p}$ is $0.05339 \mathrm{~m}^{2}$. It can be concluded from the formula that the heat flux distribution on the disc surface is related to the radial distribution of the circumferential contact length during braking.

The thermal radiation model of thermal analysis is highly non-linear, and it is difficult to obtain convergent solution in the process of finite element solution. According to Newton's cooling law, the relationship between the rate of heat dissipation by thermal radiation and the temperature difference is as follows.

$$
q=\frac{d Q}{d t}=k A\left(T-T_{0}\right)
$$

In the formula, dQ is the heat dissipated in $\mathrm{dt}$ time, W. A is the surface area of the radiation surface, $\mathrm{m}^{2}$. Then the following formula is obtained.

$$
k=\varepsilon \sigma\left(T^{2}+T_{0}^{2}\right)\left(T+T_{0}\right)
$$


Three nodes with different radial positions on the disk and four nodes with different axial positions at the agreed radius are selected. Figure 2 reflects the relationship between the node temperature of radial and axial position on the brake disc and time.

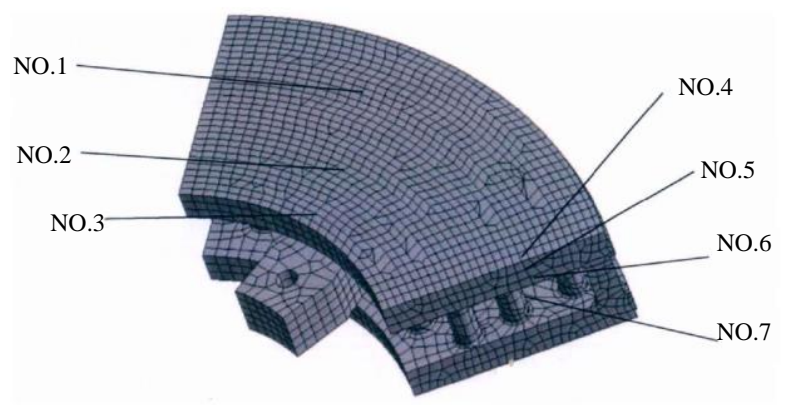

Figure 2: Selection and numbering of temperature nodes

In order to facilitate the finite element solution, according to Newton' s cooling law and Stephen Boltzmann equation, the thermal radiation coefficient can be converted into convective heat transfer coefficient as follows.

$$
\alpha_{r}=k=\varepsilon \sigma\left(T^{2}+T_{0}^{2}\right)\left(T+T_{0}\right)
$$

In the formula, $\mathrm{T}$ is the boundary temperature of the brake disc, $K . \mathrm{T}_{0}$ is the temperature of the surrounding flowing gas, $\mathrm{K}$.

\section{Results and Discussion}

\subsection{Simulation analysis of heat flux and temperature field determined by energy conversion algorithm}

From figure 3, it can be seen that the temperature at different positions along the axis of the brake disc varies with time by analyzing the data obtained. From the time history curves of the brake disc at different positions along the axis, it can be seen that the brake disc rotates faster at the beginning of friction braking, and a large amount of heat generated by the friction between the brake disc and its surface is first absorbed by the surface.

Therefore, the temperature on the surface of the brake disc rises rapidly at the beginning stage and transfers heat to the inner part of the disc, and the temperature inside the brake disc begins to rise slowly with the speed.

As a result of the factors of heat convection and heat transfer, the temperature on the surface of the disc begins to decrease, while there is a large temperature difference between the inner and the surface of the disc, which continues to absorb the heat on the surface of the disc.

The temperature increases throughout the braking process.
The closer to the surface of the brake disc is, the faster the rising speed is.

From the beginning to the end of braking, the axial temperature gradient of the brake disc decreases gradually.

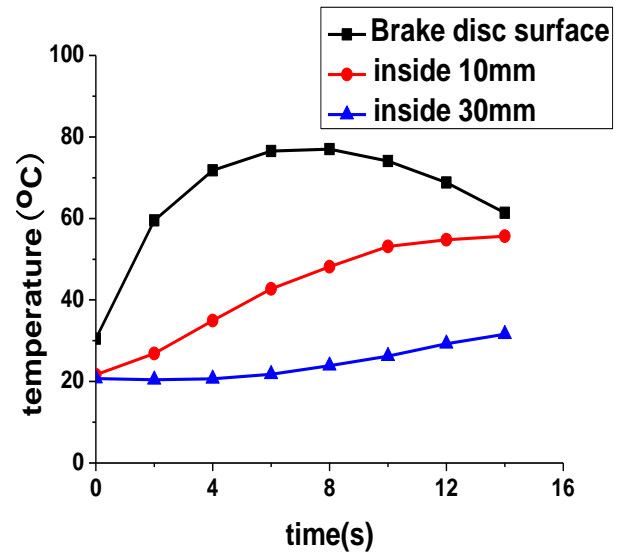

Figure 3: Temperature curve of brake disc at different axial positions with time

\subsection{Simulation analysis of heat flux and temperature field of brake disc}

Figure 4 shows that by analyzing the data obtained, the relationship between the node temperatures at different radial and axial positions on the brake disc and the time is obtained.

From figure $4 \mathrm{~A}$, it can be seen that the temperature of each node on the disk surface varies with the circumferential contact length at different radius, which leads to the radial temperature difference.

At the initial braking time, the surface temperature of the area with the greatest circumferential contact of the brake pad rises sharply, and the rate of temperature rise in each area is related to the length of circumferential contact.

With the braking process proceeding, the input heat flux density on the friction surface is lower than the heat conduction and convective heat dissipation rate, and the temperature of each node on the surface begins to decrease.

However, the inner circumferential contact length of the brake disc is small. After braking starts, the inner temperature rises slowly, and the temperature is obviously lower than that of other places.

From figure 4B, it can be seen that the temperature difference in the axial direction is due to the internal heat conduction of the disc. Heat transfers from the inside of the disk.

The temperature of the disk rises first and then decreases. The temperature inside the disk keeps rising. The trend of temperature change along the axis is similar to that calculated by the energy conversion method. 


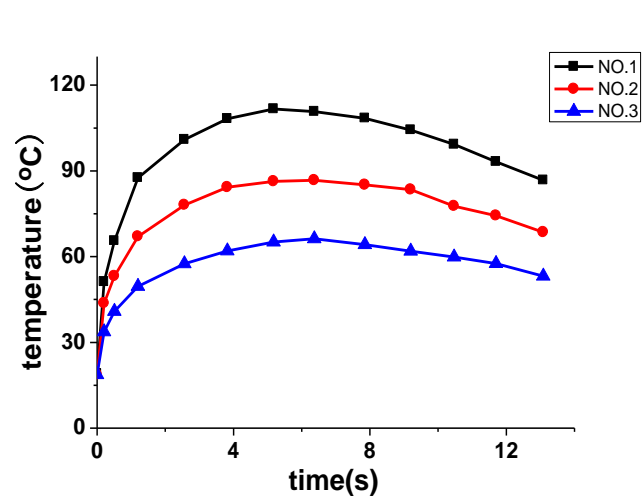

A

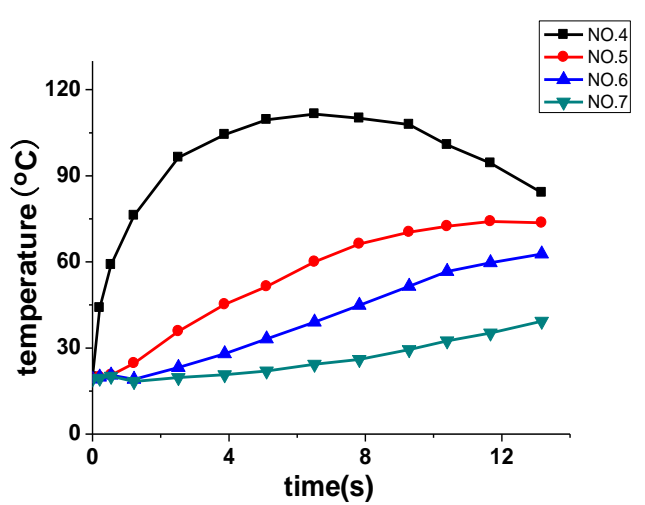

B

Figure 4: Temperature-time curves at different locations

(A. Radial different positions; B. Axial different positions)
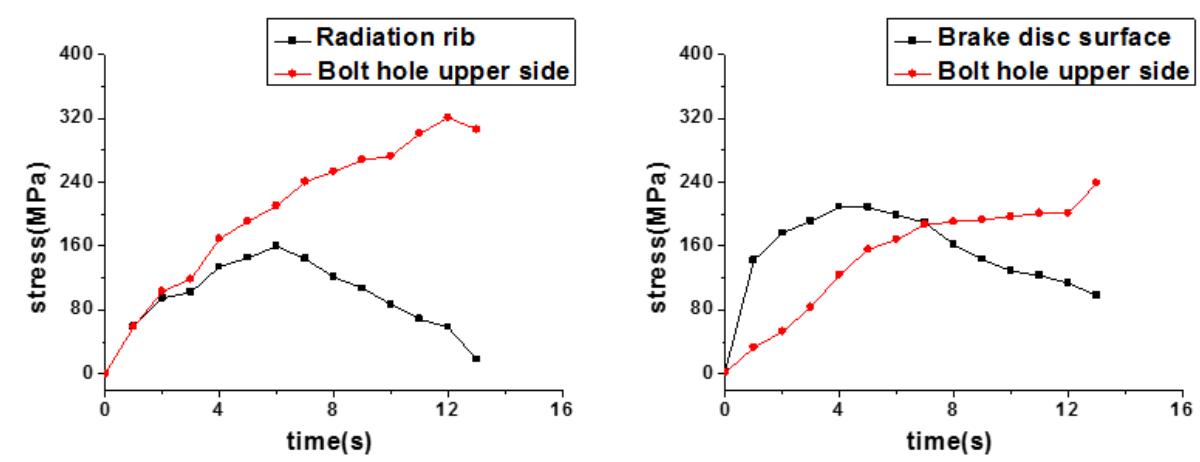

A

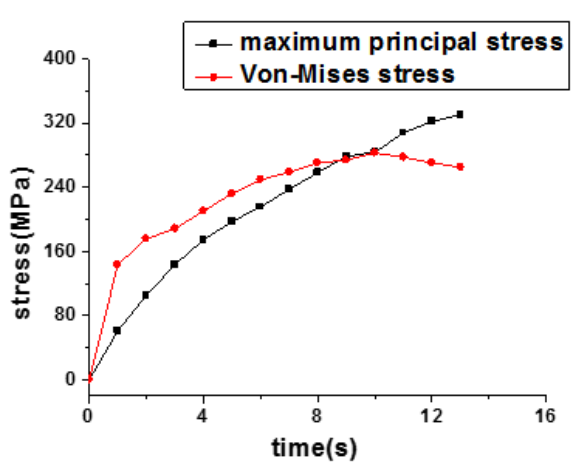

B

Figure. 5 Stress analysis diagram of finite element model

(A. Maximum principal stress on the upper side of radiating rib and bolt hole;

$B$. Von-Mises equivalent stress on friction surface and upper side of bolt hole;

C. Relation diagram of Von-Mises stress and maximum principal stress of brake disc with time)

\subsection{Analysis of thermal stress finite element model}

From figure 5 , it can be seen that the data obtained are analyzed. From figure 5A, it can be seen that the tensile stress at the radiator rib increases first and then decreases, and the maximum value reaches $160 \mathrm{MPa}$.

The tension stress in the small area on the upper side of the bolt hole increases continuously, but the increment decreases gradually, reaching the maximum value of $320 \mathrm{Mpa}$ near the end of braking. According to figure $5 \mathrm{~B}$, the Mises stress on the surface of the brake disc increases first and then decreases, and the maximum Mises stress is 210Mpa. The Mises stress in the upper part of the bolt hole increases continuously, and the maximum value is 238Mpa. Figure 5C shows that the maximum principal stress and Von-Mises stress are comprehensively analyzed. The tensile stress and equivalent stress in the upper part of the bolt hole of the brake disc teeth are the largest parts, which are most vulnerable to damage. Therefore, the results show that the stress on the upper side of the bolt hole of the brake disc teeth is the greatest during braking process, which is prone to damage. 


\section{Conclusion}

In order to study the active roll control of the brake disc bolt load of heavy semi-trailer, the thermal coupling simulation model of the brake disc of the vehicle is carried out, and then the temperature field of the brake disc is calculated by the energy folding algorithm and the friction power method combined with the principle of friction braking and heat transfer of the vehicle. The thermal stress bolt load of the brake disc is analyzed.

The results show that the temperature gradient along the axis of the brake disc decreases gradually from the beginning to the end of the braking process.

When analyzing the internal temperature of the brake disc, the heat transfers from the disc to the inside, the temperature on the disc increases first and then decreases, and the temperature inside increases continuously.

When analyzing the finite element model of the brake disc, it is found that during the braking process, the stress on the upper side of the bolt hole in the tooth part of the brake disc is the largest, which is prone to damage.

In summary, through the research on subjective roll control of heavy semi-trailer brake disc bolt load, it is found that the simulation results of active roll control of bolt load are in line with expectations, which provides a basis for the improvement of heavy semi-trailer. However, there are also some shortcomings in this research. For example, when the time variation of the data is studied, the sample size is too small. Therefore, further studies will continue to be carried out.

\section{Acknowledgement}

The Youth Key Teachers Training Program of Henan Higher Education Institutions in 2014, Item number : 2014GGJS-165

\section{References}

[1] Sripad S., Viswanathan V. (2017) Performance metrics required of next-generation batteries to make a practical electric semi truck. ACS Energy Letters, 2(7), 1669-1673.
[2] Diba F., Esmailzadeh E. (2018) Development of hybrid electric heavy-duty truck with selfpropelled trailer. International Journal of Heavy Vehicle Systems, 25(2), 203-222.

[3] Mareev I., Becker J., Sauer D. (2017) Battery dimensioning and life cycle costs analysis for a heavy-duty truck considering the requirements of long-haul transportation. Energies, 1(1), 55.

[4] Abdelkareem M. A. A., Makrahy M. M., Abd-ElTawwab A. M, et al. (2018) An analytical study of the performance indices of articulated truck semi-trailer during three different cases to improve the driver comfort. Proceedings of the Institution of Mechanical Engineers, Part K: Journal of Multi-body Dynamics, 232(1), 84-102.

[5] Saari S., Karjalainen P., Ntziachristos L, et al. (201 6) Exhaust particle and NOx emission performanc e of an SCR heavy duty truck operating in real-wo rld conditions. Atmospheric environment, 126, 13 6-144.

[6] Le V. Q. (2017) Comparing the performance of suspension system of semi-trailer truck with two air suspension systems. Vibroengineering PROCEDIA, 14, 220-226.

[7] Liem N. V., Jianrun Z., Quynh L. V, et al. (2016) Stu dy of fuzzy control for cab's isolation system of he avy truck. Vibroengineering PROCEDIA, 10, 309-3 14.

[8] Rao V. N., Eischen J. W. (2016) Failure analysis of mixed mode crack growth in heavy duty truck frame rail. Case Studies in Engineering Failure Analysis, 5, 67-74.

[9] Lowrie J., Pang H., Ngaile G. (2017) 0Weight reduction of heavy-duty truck components through hollow geometry and intensive quenching. Journal of Manufacturing Processes, 28, 523-530.

[10] Aliakbari K., Imanparast M., Masoudi Nejad R. (2018) Microstructure and fatigue fracture mechanism for a heavy-duty truck diesel engine crankshaft. Sci Iran, 1939.

[11] Godbole S., Lam N., Mafas M, et al. (2018) Dynamic loading on a prefabricated modular unit of a building during road transportation. Journal of Building Engineering, 18, 260-269. 